\title{
Nurse's Roles and the Mediating Effects of Stress on Job Performance in Low and Developing Economies
}

\author{
Joshua Chiroma Gandi ${ }^{1}$, Wukatda Wokji Beben ${ }^{2}$, Yohanna Gyarazama ${ }^{2}$ \\ ${ }^{1}$ Department of General and Applied Psychology, University of Jos, Jos, Nigeria; \\ ${ }^{2}$ School of Nursing, Ministry of Health, Jos, Nigeria. \\ Email: jcgandi2000@yahoo.com, gandij@unijos.edu.ng \\ Received March 16 ${ }^{\text {th }}, 2011$; revised May 13 ${ }^{\text {th }}, 2011$; accepted June $1^{\text {st }}, 2011$.
}

\begin{abstract}
The role of nursing is observed to have been associated with multiple and conflicting demands. This study was designed to examine nurse's roles and the mediating effects of stress on job performance in low and developing economies. Participants were hospital-based Nurses $(n=373)$ measured with "Maslach Burnout InventoryGeneral Survey, Job Autonomy Questionnaire, Questionnaire on Organizational Stress-Doetinchem and Job Diagnostic Survey". The role of work-home interference (WHI) and home-work interference (HWI) with respect to work characteristics, burnout, and gender were analyzed, using SPSS 15.01 version. No gender differences in burnout levels; WHI and HWI were found to mediate the relationship between work characteristics and burnout. Lack of significant gender difference regarding burnout might be due to the fact that men and women in Nigeria have similar working conditions. High workload and home roles seem contributory to high levels of exhaustion. Evidence indicate the need to lower the pace of work and number of patients per Nurse, to decrease workload thereby reducing emotional exhaustion. There is sufficient evidence to believe that work stress is a factor among health care personnel. To derive a better understanding of stress and burnout in the workplace, studies need to move beyond the tendency of using descriptive designs. Such studies would aid understanding the effect of stress and burnout on patient safety (outcomes).
\end{abstract}

Keywords: Gender, Home-Work Interference (HWI), Job Performance, Nurse's Roles, Nursing, Work-Home Interference (WHI), Stress

\section{Background to the Study}

In terms of broad behavioural construct, the general taxonomy of job performance includes "assessment of performance, analysis of job, and method of job elements" (Dokotri, 2006). A model of job performance which takes into account the multifaceted nature of job and separates the various elements subsumed under that rubric makes fundamental distinctions. Thus, distinctions between: aspects of work evaluation that are under the control of the worker (behaviours involved in job performance itself), aspects not under the control of the worker (the consequence or effectiveness of job performance), aspects that deals with relative costs (productivity), and aspects that showed the value placed on each by the organization (utility). The workers' behaviour, job effectiveness, productivity, and utility have inherent consequences. Hence, factors capable of inducing stress might include catastrophes, major life changes, and daily hassles, among others (Wai, 2009). Work-related stress can impact on an organization based on the context (the organizational culture and function) and content (the work environment and equipment). This study was premised on the concepts: "job performance, nursing roles, stress' as the variables of interest.

\section{Conceptual Framework}

\section{Nurse's Roles}

The word "role" is a term borrowed by social scientists from drama. In its theatrical context, role refers to a person pretending to be someone else for the purposes of entertainment. This implies certain deception and that is not the way the word "role" is used in the phrase the "nurse's role". It is acceptable to say that "role" is the term applied to human behaviour, based on the fact that human being behave in certain relatively predictable patterns (Wai, 2009). Role is a descriptive word for relatively predictable behavioural patterns. Wai (2009) inferred that the nursing role, then, refers to all behaviours that are considered appropriate for a nurse. Going down memory lane, the nurse's role could be described in terms of the historical images of the nurse. Kato (1994) identifies three historically traditional images that still influence the nursing profession:

The Folk Image: The folk image of the nurse as "mother" arises from the original use of the word "nurse" to mean suckling the young. This meaning quickly broadened to that of caring for the sick and the aged. Such care was provided by simple methods passed on from one person to another. The folk image, which is still held in some quarters today, is an emotional view of the nurse as "mother: gentle, kind, always available, nurturing life by natural means, wise but not learned.

The Religious Image: The care of the sick has always been seen as a Christian duty in Western civilization. Thus, the Church viewed caring for the sick as important for the salvation of the soul of the care-giver. Over the centuries, this religious image reinforced the characteristics of the folk image, since it suggested that nursing should be done for love and required no formal learning. Other notions were the beliefs that a nurse should be celibate, cloistered, unworldly and strictly disciplined. 
The Servant Image: This image arose during the 16th to the 19 th centuries, been the dark ages in nursing history. During this time, illness was seen as a punishment for sin and the care, if any, given to the sick was far from charitable. Any nursing that was available generally was given by ill-paid, ignorant and, sometimes, immoral women.

Nurse's Preparation for Roles: Roles, however, have to be learned by the instrumentality of socialization which occurs in intentional learning and or incidental learning. A nurse is socialized intentionally through the formalized education and experiences received during training programmes. Incidental learning or socialization occurs through more casual interactions within the health care system and other relevant outfits. Nursing is synonymous with caring which encompasses empathy for and connection with people. Despite the expanded roles (practitioner, clinician, clinical specialist and others), the professional nurse is at times in situations that pose ethical and or moral conflict.

Stress: Throughout its history as a psychological construct, stress has proven to be relatively complicated and has inspired confusion. Conceptualized, at a point, according to mechanical principles of load (external force) or the area upon which that force is exerted, stress "signals danger" and "prepares" us to take defensive action. For instance, the fear of things that pose realistic threats motivates us to deal with them or avoid them. Moderate stress fuels creativity and motivates us to achieve. But excessive stress leads to less cooperation, more aggression, and even hinders performance on difficult tasks. Psychologists view stress in three ways, including as: 1) a stimulus, 2) a response, and 3) an ongoing interaction between the organism and its environment.

Factors capable of inducing stress have been described in terms of catastrophes (calamities or disasters), major life changes (bereavement, poverty, loss of possession, illness/disability) and daily hassles (academic pursuits, work-related issues, relational problems or unfriendly environment). Work-related stress can impact in many ways, including the context (organisational culture and function) and the content (work environment and equipment). The commonly accepted definition reads "stress is a condition or feeling experienced when a person percieves that demands exceed the personal and social resources the individual is able to mobilize" (Lazarus, 1984). Thus, stress is what we experience when we feel that we are not in control. Work stress refers to the harmful physical and emotional responses that occur when job requirements do not match the worker's capabilities, resources, and needs (Murphy \& Sauter, 1999).

\section{Statement of Problem}

Until now, not much research has been conducted on nurse's roles in relation to work characteristics and family responsibility on the employees' performance in low income and developing countries like Nigeria. Considering that individuals in developing economies experience continuous and prolong stress (such as those related to political instability, civil unrest, and resource mis-management among others), it is particularly useful to see how these citizens (especially caring professionals like Nurses) cope in terms of work and stress. Moreso, Wai (2009) observed that Nursing Profession has not been openly appreciated in Nigeria despite her positive impacts on the lives of Nigerians and the public at large.

\section{Significance of the Study}

This study would be of importance to health care providers, researchers, health agencies, regulatory bodies and serviceusers (the patients). It would serve as the means through which capacity of the caring professionals could be built for more effective job performance. The outcome is a veritable tool that could serve as a wake-up call to enhance capacity building for effective caring services. It would provide more impetus for the quality improvement and evidence-based goal achievement being pursued. Regulatory bodies would be more informed when formulating policies or determining the necessary conditions for professional practice. Healthcare agencies (public or private), who are proprietors of health service outfits, would benefit from the study results as empirical evidence (as claim) that could enhance their management decisions and supervisory roles.

\section{Purpose and Objectives}

\section{Purpose of the Study}

The main purpose of this study was to investigate nurse's roles and the mediating effects of stress on job performance among in low and developing economies of the world.

\section{Objectives of the Study}

The research aims at achieving the following objectives:

1) To assess the effects of stress among male and female Nurses in Nigeria

2) To determine direct relationships (if any) between work characteristics, gender and Burnout

3) To investigate the role of work-home and home-work interferences on job performance.

\section{Study Hypotheses}

The study hypothesized that:

(H1) stress and burnout prevalence among Nurses in the low income and developing economies is high due to high workload;

(H2) women score higher on emotional exhaustion, while men score higher on depersonalization;

(H3) job demands are primarily related to emotional exhausttion, while job resources are primarily related to depersonalizetion and personal accomplishment;

(H4) both WHI and HWI are higher for women than men;

(H5) both WHI and HWI mediate the relationship between work characteristics and burnout outcomes; and this mediational effect is stronger for women.

\section{Methodological Approach and Justification}

\section{Study Setting \& Population}

The study was conducted in Bauchi State, under the State Chapter of the National Association of Nigerian Nurses \& 
Midwives (NANNM). Situated in the northeast zone, Bauchi State is one of the 36 states of the Federal Republic of Nigeria. The state, which housed a good number of hospitals/health centers, have three Senatorial Districts and 16 Local Government Areas. These hospitals/health centers are variously proprietored by Federal Government, State Government, Local Governments, Companies/Corporate Organizations, and private enterprenures. During the period of this study, Bauchi State population stood at 4.1 million people. There were then 3698 Nurses, including those under the services of Federal Government, State Government, Local Governments, Companies/ Corporate Organizations, and private hospitals/clinics. These Nurses consisted of male and female as well as Christians and Muslims.

\section{Participants and Sampling Techniques}

From the 3698 Nurses, 373 study participants were selected by stratified random sampling. The stratification technique included geographical location (senatorial zones) of the hospitals/health centers and sex (or gender) of the Nurses. Among the 373 participants, 113 were randomly sampled from Bauchi North senatorial zone, 100 from Bauchi central senatorial zone and 160 from Bauchi South senatorial zone. Overall, the participants included 99 male muslims, 85 male christians, 83 female muslims and 98 female christians. The sampling technique made use of formal identifying information, not the physical Nurses. Access to the staff inventory, containing demographic and personal data of prospective participants, at each participating hospital/health center's personnel office have reasonably facilitated the whole sampling exercise.

\section{Design}

This research was an exploratory (survey-type) design. "Nurse's roles" and the mediating "effects of stress" was considered the independent variables while "job performance" considered as the dependent variable.

\section{Instruments and Measures}

1) Maslach Burnout Inventory-General Survey (Maslach \& Leiter, 1997): Burnout was measured using the Maslach Burnout Inventory-General Survey (Maslach \& Leiter, 1997) which contained 22 items and three scales. Emotional exhaustion was measured by ten items $(\alpha=.89)$, depersonalization by five items $(\alpha=.68)$ and reduced personal accomplishment by seven items $(\alpha=.75)$. Previous research shows that the reliability of depersonalization score is usually low. Responses were made on a six-point scale ( $0=$ never, $6=$ every day $)$.

2) Job Autonomy Questionnaire (De Jonge, Landeweerd, \& Nijhuis, 1993): Workload was measured by an eight item scale $(\alpha=.87)$ based on the Job Autonomy Questionnaire (De Jonge, Landeweerd, \& Nijhuis, 1993). The participants indicated their agreement with each item on a four-point scale $(1=$ never, $4=$ very often). Job control was measured by 4 items $(\alpha=.72)$ of the Inventory of feelings of motivation and demotivation. Responses were made on a four point scale $(1=$ not at all, $4=$ very much). Work-home and home-work interference were assessed via two scales consisting of 13 items. Work-home interference was measured by seven items $(\alpha=.90)$. Home-work interfereence was assessed by six items $(\alpha=.84)$. The respondents an- swered via five point scale $(1=$ never, $5=$ always $)$.

3) Questionnaire on Organizational Stress-Doetinchem (VOS$D)$ : Social support was measured by three 5 -item scales deducted from the Questionnaire on Organizational Stress-Doetinchem (VOS-D), each assessing the social support received from supervisor/colleague, partner and family/friends. The scores on three subscales were averaged to yield a sum score. Reliability of the scale was good $(\alpha=.82)$. Items were rated on four point scales ( 1 = never, 4 = always).

4) Job Diagnostic Survey (Hackman \& Oldham, 1980): Work content was assessed via five items based on the Job Diagnostic Survey (Hackman \& Oldham, 1980. The scale $(\alpha$ $=.82)$ was based on a four point answering options $(1=$ never, $4=$ very often).

\section{Data Collection}

\section{Methods}

Field Assistants: Research field assistants $(n=12)$ were recruited and given appropriate orientation on effective administration and retrieval of questionnaires.

Procedure: Data collection procedure commenced after observing the appropriate tenets and protocols that defined ethical consideration. The following sequence of activities then took turns:

- Appropriate administration of instruments (questionnaires) to the participants

- Reetrieval/collection of all completed instruments (questionnaires).

- Collation of the retrieved questionnaires for onward analyses.

\section{Ethical Issues and Approval}

Institutional approvals were sought from and granted by Education Standards and Research Committee of the National Association of Nigeria Nurses \& Midwives (Bauchi State Chapter). All participants individually signed an informed consent for participation and were duly debriefed after the exercise.

\section{Data Analysis}

In order to determine gender differences in the level of burnout, "independent samples" $t$-tests were performed. The cut-off points for different levels of burnout were obtained from the Utrechtse Burnout Schaal (UBOS) manual (Schaufeli \& Dierendonk, 2000). The relationship between work characteristics and burnout was analyzed by means of linear hierarchical regression analyses. Because of high multicollinearity of WHI and HWI, separate analyses were carried out for these variables. The mediating role of WHI and HWI was assessed using the recommended method of analyses in line with Mackinnon, Lockwood \& Hoffman (2002); by checking whether paths from predictor to intervening variable and from intervening variable to outcome variable are both significant. If so, then the intervening variable is a mediator of the relationship. This method has most power and most accurate Type 1 error rates as compared to other mediation tests (Mackinnon, Lockwood, \& Hoffman, 2002). All the analyses were performed using SPSS 15.01 version. 


\section{Results/Findings}

\section{Preliminary Analyses and Burnout Prevalence among Nurses}

Emotional exhaustion experienced by Nigerian Nurses was medium to high and depersonalization was medium. At the same time, they felt they were doing their job very well, having on average high levels of personal accomplishment. Thus, the first hypothesis was confirmed partially (see Table 1). The second hypothesis regarding gender differences and burnout was not confirmed, since no significant gender differences were detected. The trend was however, that females experienced more emotional exhaustion, higher depersonalization and higher personal accomplishment than men.

\section{Direct Relationships between Work Characteristics, Gender and Burnout}

The predicted stronger relationship between job demands than job resources on emotional exhaustion (H3) was confirmed only for women. For men, job resources and job demands play an equally important role in determining levels of emotional exhaustion (see Table 2). The predicted stronger relationship between job resources than job demands on depersonalization (H3) was confirmed only for men. For women both job demands and job resources equally contribute to depersonalization. For men, variance accounted for by job characteristics is higher than for women, suggesting that other variables play a more important role in predicting depersonalization in women. Predicted stronger relationship between job resources than job demands on personal accomplishment (H3) was confirmed for both men and women. However, for men, work characteristics accounted for greater portion of variance (19\%) than for women $(8 \%)$. This implies that for women other variables than work characteristics are more important in accounting for levels of personal accomplishment.

\section{Role of Work-Home and Home-Work Interference}

The fourth hypothesis about higher levels of WHI/HWI among women could not be confirmed. No statistically significant gender differences regarding overall levels of WHI/HWI were found. Mean levels of WHI were higher than HWI levels for both genders. Subanalyses (not shown) revealed that $20.7 \%$ of men and $30.8 \%$ of women experienced WHI at least occasionally, while the same analyses for HWI revealed the prevalence of $5.2 \%$ for men and $5.1 \%$ for women.

The results regarding mediating effects of WHI and HWI between work characteristics and burnout outcomes are presented in Figure 2. For men, WHI mediates the relationship between work characteristics and emotional exhaustion. Strongest positive association of work home interference was with job demands and negative association with job control. No mediating role was found regarding depersonalization and personal accomplishment. For women, WHI mediates the relationship between work characteristics and emotional exhaustion and depersonalization. Strongest positive association found was between job demands and WHI, and strongest negative association was between job control and WHI. No mediating role of WHI between work characteristics and personal accomplishment was found.

In both male and female Nurses, HWI mediates the relationship between work characteristics and all three burnout variables. For men, lack of social support is most strongly related to HWI, which in turn has the strongest association with emotional exhaustion. The same pattern is found among female Nurses, but the strength of the relationship is weake Thus, the

Table 1.

Descriptive Characteristics and Correlations between variables for males $(N=56$ to 58$)$ in the top right corner and females $(N=308$ to 315$)$ in the bottom left corner.

\begin{tabular}{|c|c|c|c|c|c|c|c|c|c|c|c|c|c|c|c|}
\hline Variables & Mean & SD & Mean & SD & t-test & 1 & 2 & 3 & 4 & 5 & 6 & 7 & 8 & 9 & 10 \\
\hline Age & 47.43 & 10.16 & 47.38 & 8.48 & .03 & 1 & -.01 & $-.22 * *$ & .03 & -.04 & -.02 & $.14 * *$ & $.11 *$ & .01 & -.05 \\
\hline $\begin{array}{l}\text { Emotional } \\
\text { exhaustion }\end{array}$ & 2.25 & 1.33 & 2.51 & 1.30 & 1.42 & -.01 & 1 & $.44 * *$ & -.12 & $.60 * *$ & $-23 * *$ & $-.21 * *$ & $.27 * *$ & $.56^{* *}$ & $.33 * *$ \\
\hline $\begin{array}{l}\text { Depersona } \\
\text { lization }\end{array}$ & .63 & .65 & .75 & .92 & .75 & $-.22 * *$ & $44 * *$ & 1 & $-.24 * *$ & $-27 * *$ & -.07 & $-.19 * *$ & $.18^{* *}$ & $.29 * *$ & $.34 * *$ \\
\hline $\begin{array}{c}\text { Personal } \\
\text { accomplishment }\end{array}$ & 5.10 & 1.06 & 5.18 & .81 & -.63 & .03 & $-.12 *$ & $-.24 * *$ & 1 & -.04 & $.19^{* *}$ & $.31 * *$ & $-.18 * *$ & -.05 & $-.21 * *$ \\
\hline $\begin{array}{c}\text { Job } \\
\text { demands }\end{array}$ & 2.83 & .58 & 2.93 & .60 & -.17 & -.04 & $.60 * *$ & $.27 * *$ & -.04 & 1 & $-.14 * *$ & -.08 & $.13 * *$ & $.56^{* *}$ & $.29 * *$ \\
\hline Job control & 2.89 & .76 & 2.74 & .66 & 1.54 & -.02 & $-.23 * *$ & -.07 & $.19^{* *}$ & -.14 & 1 & $.34 * *$ & $-.30^{*}$ & $-.23 * *$ & $-.17 * *$ \\
\hline Job content & 2.89 & .73 & 2.92 & .58 & -.34 & $.14 * *$ & $-.21 * *$ & $-.19 * *$ & $.31 * *$ & -.08 & $.34 * *$ & 1 & $-.32 * *$ & $-.15^{*}$ & $-.19 * *$ \\
\hline $\begin{array}{l}\text { Work on home } \\
\text { interference }\end{array}$ & 2.42 & .85 & 2.58 & .80 & -1.35 & -.01 & $.56^{* *}$ & $.30 * *$ & -.05 & $.56^{* *}$ & $-.23 * *$ & $-.15 * *$ & $.27 * *$ & 1 & $.59 * *$ \\
\hline $\begin{array}{l}\text { Home on work } \\
\text { interference }\end{array}$ & 1.65 & .58 & 1.68 & .61 & -.44 & -.05 & $.33 * *$ & $.34 * *$ & $-.21 * *$ & $.29^{* *}$ & $-.17 * *$ & $-.19 * *$ & $.34 * *$ & $.59 * *$ & 1 \\
\hline
\end{tabular}

Note: $* * \mathrm{p} \leq 0.01$ level (2-tailed); ${ }^{*} \mathrm{p} \leq 0.05$ level ( 2 -tailed) missing values were handled by pairwise deletion. 
Table 2.

Means, standard deviations, and correlations between the model variables.

\begin{tabular}{|c|c|c|c|c|c|c|c|c|c|c|c|c|}
\hline \multicolumn{13}{|c|}{ Males $(N=184)$} \\
\hline Variable & $M$ & $S D$ & 1 & 2 & 3 & 4 & 5 & 6 & 7 & 8 & 9 & 10 \\
\hline 1. WHI & 2.15 & 0.81 & - & & & & & & & & & \\
\hline 2. HWI & 1.46 & 0.63 & .31 & - & & & & & & & & \\
\hline 3. Quantitative job demands & 2.52 & 0.82 & .39 & .12 & - & & & & & & & \\
\hline 4. Emotional job Demands & 1.71 & 0.71 & .29 & .17 & .46 & - & & & & & & \\
\hline 5. Mental job Demands & 2.97 & 0.82 & .22 & .14 & .63 & .48 & - & & & & & \\
\hline 6. Quantitative home demands & 2.26 & 0.78 & .21 & .27 & .32 & .30 & .39 & - & & & & \\
\hline 7. Emotional home Demands & 1.66 & 0.65 & .23 & .42 & .31 & .35 & .40 & .49 & - & & & \\
\hline 8. Mental home Demands & 1.89 & 0.76 & .16 & .30 & .30 & .27 & .41 & .69 & .55 & - & & \\
\hline 9. Exhaustion & 2.78 & 1.11 & .40 & .34 & .55 & .60 & .50 & .39 & .40 & .34 & - & \\
\hline 10. Cynicism & 2.49 & 1.19 & .22 & .33 & .22 & .30 & .23 & .31 & .30 & .28 & .64 & - \\
\hline
\end{tabular}

Note: All correlations are significant at the $\mathrm{p}<.01$ level.

\begin{tabular}{|c|c|c|c|c|c|c|c|c|c|c|c|c|}
\hline \multicolumn{13}{|c|}{ Females $(N=184)$} \\
\hline Variable & $M$ & $S D$ & 1 & 2 & 3 & 4 & 5 & 6 & 7 & 8 & 9 & 10 \\
\hline 1. WHI & 2.15 & 0.81 & - & & & & & & & & & \\
\hline 2. HWI & 1.46 & 0.63 & .31 & - & & & & & & & & \\
\hline 3. Quantitative job demands & 2.52 & 0.82 & .39 & .12 & - & & & & & & & \\
\hline 4. Emotional job Demands & 1.71 & 0.71 & .29 & .17 & .46 & - & & & & & & \\
\hline 5. Mental job Demands & 2.97 & 0.82 & .22 & .14 & .63 & .48 & - & & & & & \\
\hline 6. Quantitative home demands & 2.26 & 0.78 & .21 & .27 & .32 & .30 & .39 & - & & & & \\
\hline 7. Emotional home Demands & 1.66 & 0.65 & .23 & .42 & .31 & .35 & .40 & .49 & - & & & \\
\hline 8. Mental home Demands & 1.89 & 0.76 & .16 & .30 & .30 & .27 & .41 & .49 & .45 & - & & \\
\hline 9. Exhaustion & 2.78 & 1.11 & .50 & .34 & .45 & .45 & .40 & .69 & .55 & .46 & - & \\
\hline 10. Cynicism & 2.49 & 1.19 & .22 & .33 & .22 & .30 & .23 & .31 & .30 & .28 & .64 & - \\
\hline
\end{tabular}

Note: All correlations are significant at the $\mathrm{p}<.01$ level.

fifth hypothesis was confirmed partially. WHI and HWI are mediating the relationship between job characteristics and some burnout outcomes, but the relationship is stronger for men than women.

\section{Limitations of the Study}

This study has a number of limitations. Considering that we used a cross sectional method of data collection, no causal relationships could be established and no insight is available into how the situation has been developing from then on.

\section{Discussion}

This study assessed nurse's roles and the mediating effects of stress on job performance which have not been sufficiently explored in low and developing economies of the world. Hence, the role of work-home and home-work interferences were also examined with respect to work characteristics while paying special attention to gender.

\section{Burnout Prevalence and Gender Differences among Nurses}

The results regarding burnout and stress could be explained in terms of JD-R model. Since the levels of job demands among Nurses were high (see Table 1), this was reflected in exhaustion from work. However, job resources were also high, which buffered this negative role of job demands (Bakker, Demerouti, $\&$ De Boer, 2003). Lack of significant gender difference regarding burnout might be due to the fact that men and women 


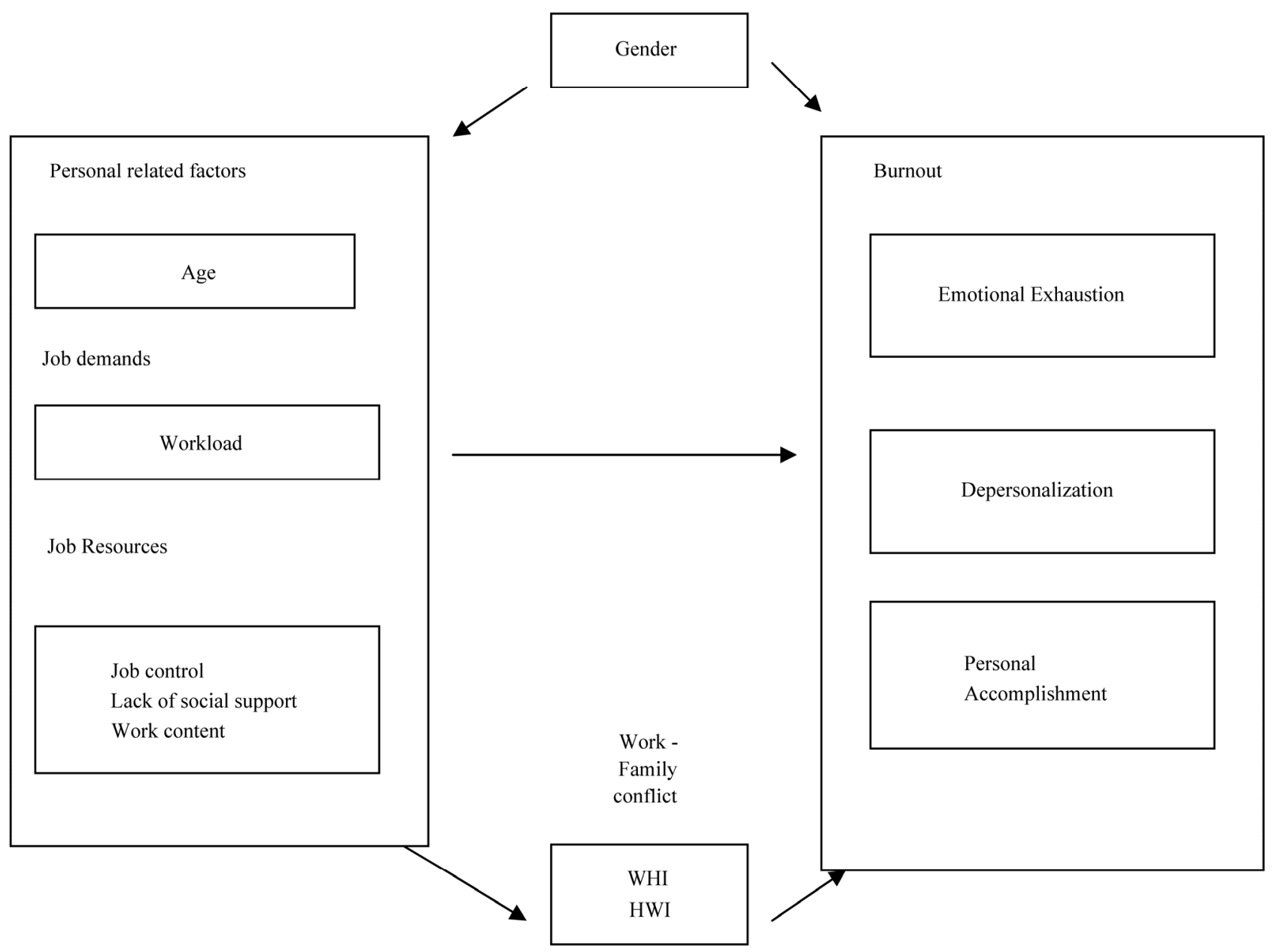

Figure 1.

The concept model of relationship between work characteristics, work-home interference/home-work interference on burnout.

in Nigeria have very similar working conditions. The Nurses' empathy for and connection with patients actually demonstrates the five core professional values which are essential but, consequently, attracts factors capable of inducing stress.

The role of nursing is associated with multiple and conflicting demands, impose by the patients' needs, which are sources of initiating and promoting burnout. An explanation for this might be in terms of high workload, having many patients (with varying needs) per Nurse. However, family and a wider socioeconomic perspective could also play a role. High levels of exhaustion experienced among Nigerian Nursess may be a reflection of accumulated tiredness from wider economic and political worries that persons carry and that spills-over to work, resulting in work-related exhaustion. Prevalence of personal accomplishment might be inherent when Nurses often see clients generally recover fast, following their caring services.

\section{Direct Relationship between "Work Characteristics and Burnout” Outcomes}

Job demands were associated with emotional exhaustion for both genders, which is in line with the JD-R model and the study hypothesis that predicts a strong association between demands and exhaustion (Bakker \& Geurts, 2004). However, for men, the link between job resources and emotional exhaustion was as important as between job demands and emotional exhaustion, which contradicts the JD-R model. Depersonalization was strongly related to job resources for men and only weakly for women. Consequently, job demands were more strongly associated with women than men. That is probably because women have more elaborate systems of support than men and so lack of emotional support regarding the work does not have much effect on them. Thus, when social support is lacking, men react by distancing themselves from patients, while women have other sources of support that buffer the lack of this type of support.

Job resources (as predicted by the JD-R model) are associated with the personal accomplishment (Bakker \& Geurts, 2004; Schaufeli \& Enzmann, 1998). There is a gender difference with regards to this component; with job resources playing a more important role in determining levels of personal accomplishment for men than women. This is in line with earlier study (Houkes, Winnants, \& Twellaar, 2008), which found different role of job resources for men and women in relation to burnout components.

It was found that levels of WHI are higher than levels of HWI. It appears that the boundary from work to home duties is 


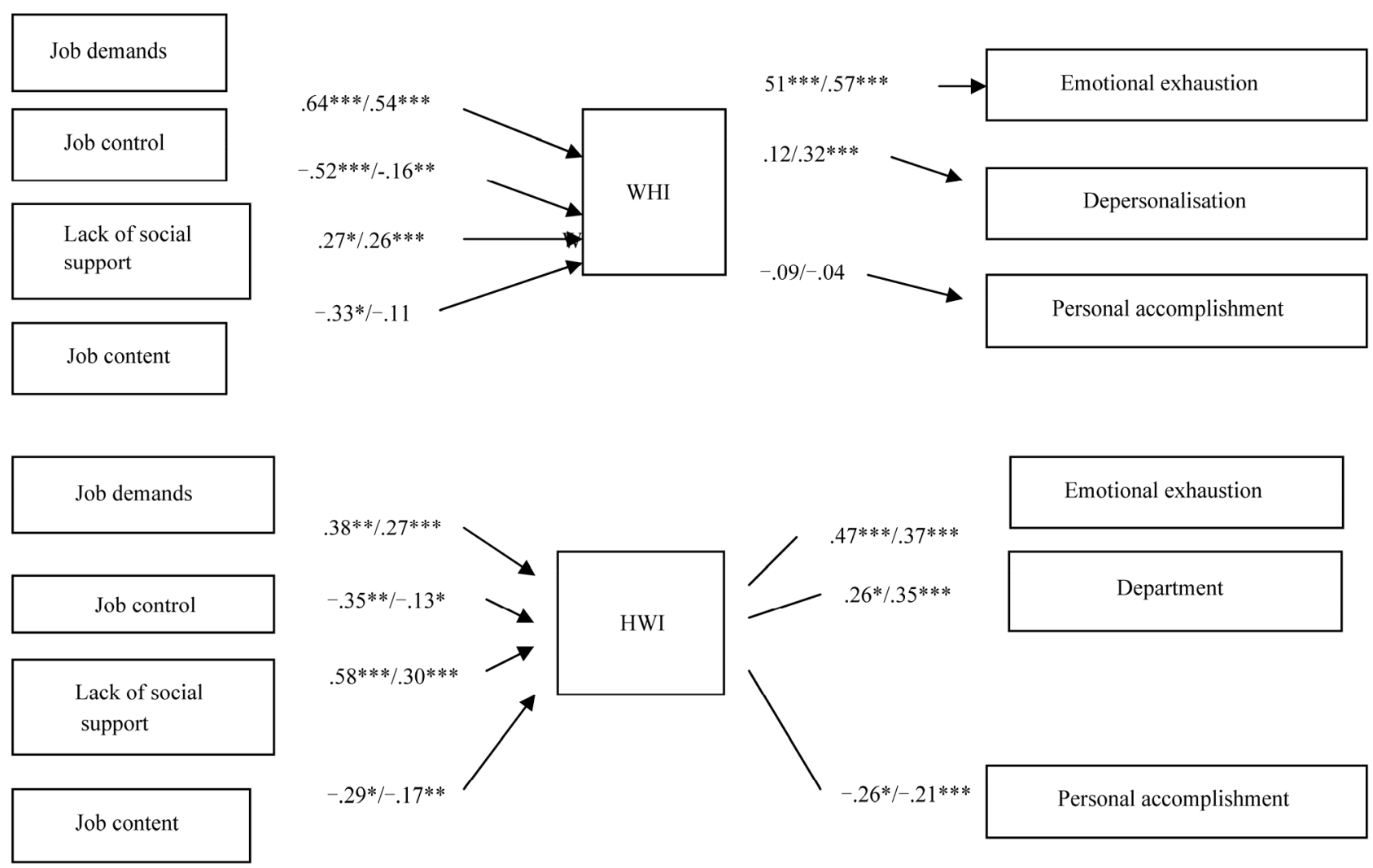

Figure 2.

Mediating role of WHI and HWI for males/females.

more permeable than the boundary from home to work. Higher WHI might be a reflection of broader societal phenomena that values career success more than family harmony. Since WHI interferes with meeting family demands, it can be particularly strenuous for women, for whom family-related self-image is often an important part of identity.

No gender differences regarding the levels of WHI/HWI were found. However, women in this study indicated being more in charge of home duties than their partners. Stemming from this, home-work interference for women would have been expected to be higher. One possible explanation might lie in women's extensive web of social network that they can rely on, which ensures that the childrearing duties do not spill-over to work. Furthermore, the fact that women did more chores at home might not lead to interference with work, but rather with free time and leisure activities. However, regarding the mediating role of WHI and HWI, some gender differences were noted. For men, the strength of relationships between predictor variables and WHI/HWI as mediators was stronger than for women. This is surprising, considering that the sample size of males was much smaller, which should have led to less power. This implies that in particular for males, WHI/HWI are important mediators of relationship between work characteristics and burnout outcomes.

\section{Implications for Research and Practice}

Results of our study indicate obvious need to lower the pace of work and number of patients per Nurse. This is crucially anticipated in order to decrease workload, which should subsequently decrease emotional exhaustion. Decreasing work demands is especially beneficial for women, since for them the demands are linked both to exhaustion and distancing from clients. At the same time, improving available resources such as better social relations and more autonomy are important (especially for male Nurses) since they are linked with all three burnout outcomes. Considering that a complex bureaucratic healthcare system is the issue, such changes need to be incurporated both in a wider policy level as well as at a micro, departmental level.

Roughly every third female and every fourth male Nurse in our sample experienced WHI at least occasionally. This group should be attended to, since WHI is directly associated in women with levels of exhaustion and distancing, and plays an important mediating role in males between work characteristics and emotional exhaustion. When WHI is low, it is a protective factor against negative work characteristics, while when it is high, it is an important risk factor. Thus in the future, particular attention should be paid to public health policy that would enhance as much as possible the healthy work-life balance.

HWI level in our sample was very low and at the moment is not an important concern for the employees. However, it is a predictor of burnout and its mediating role is very potent and should not be neglected. Should the level of HWI increase, such as due to childcare related needs or extended family caretaking duties, persons are then particularly vulnerable to the negative 
influence of job demands and lack of job resources. In this respect, no gender differences were observed and both male and female are equally vulnerable.

Gender differences in terms of contribution of job demands and job resources to burnout subcomponents call for a gender sensitive approach not only in research work, but also in development of theoretical models. It might be that different models apply for two genders. Thus in the future, it is advisable to develop and test gender specific models that will provide better understanding of gendered expressions of health (Hammarstrom, 2003).

\section{Conclusion}

Considering the perceived implications of the study findings, there's need for replication and further studies. It seems acceptable to conclude that intervention studies will, in particular, help remedy the inherent problems and or prevent them.

\section{Acknowledgements}

We thank the Bauchi State Nurses for their kind participating willingly as study participants. We also thank Dr P. S. Wai, Dr H. Karick and Dr A. Zamani for their selfless mentorship.

\section{References}

Bakker, A. B., \& Geurts, S. A. E. (2004). Toward a dual-process model of work-family interference. Journal of Work and Occupations, 31, 345-366.

Bakker, A. B., Demerouti, E., \& De Boer, E. (2003). Job demands and job resources as predictors of absence duration and frequency. Journal of Vocational Rehabilitation, 62, 341-356.

Dokotri, J. (2006). Job analysis, performance and satisfaction. Vocational Psychology Presentation. Jos: University of Jos.

Hackman, J. R., \& Oldham, G. R. (1980). Work redesign. Reading. Boston, MA: Addison-Wesley.

Hammarstrom, A. (2003). The integration of gender in medical research and education-obstacles and possibilities from a Nordic perspective. Women \& Health, 37, 121-133.

Houkes, I., Winants, Y. H. W. M., \& Twellaar, M. (2008). Specific determinants of burnout among male and female general practitioners. A cross-lagged panel analysis, 81, 249-276.

Jonge de, J., Landeweerd, J. A., \& Nijhuis, F. J. N. (1993). Constructie en valideringvan de vragenlijst ten behoeve van het project "autonomie in het werk" [Construction and validation of the questionnaire for the job autonomy project]. Studies bedrijfsgezondheidszorg, 9. Maastricht: University of Limburg.

Kato, G. U. (1994). Nursing and the nurses' role. A conference paper. Kaduna: School of Psychiatric Nursing.

Lazarus, R. S. (1984). Stress appraisal and coping. New York: Springer.

MacKinnon, D. P., Lockwood, C. M., Hoffman, J. M. (2002). A comparison of methods to test mediation and other intervening variable effects. Psychological Methods, 7, 83-104.

Schaufeli, W. B., \& Enzmann, D. (1998). The burnout companion to study and practice: A critical analysis. Philadelphia, PA: Taylor \& Francis.

Shanafelt, T. D., Bradley, K. A., Wipf, J. E., \& Back, A. L. (2002). Burnout and self-reported patient care in an internal medicine residency program. Annals of Internal Medicine, 136, 358-67.

Twellaar, M., Winants, Y., \& Houkes, I. (2008). How healthy are Dutch general practitioners? European Journal of General Practice, 14, 1-6.

Wai, P. S. (2009). Nursing profession in Nigeria. A Paper Delivered during the Taraba State Nurses' Week Program. Jalingo: Nigeria Nurses Association. 